\title{
Analysis of alarming signals for the progression of atrophic gastritis to dysplasia
}

\author{
Qiang Zhang ${ }^{1}$, Zhouyang Lian², Lei Wang ${ }^{1}$, Yadong Wang ${ }^{1}$, Fachao Zhi ${ }^{1}$, Shengli An ${ }^{3}$, Bo Jiang ${ }^{1}$, \\ Yali Zhang ${ }^{1}$ and Yang Bai ${ }^{1}$
}

${ }^{1}$ Department of Gastroenterology. Nanfang Hospital. Guangzhou, China. ${ }^{2}$ Department of Radiology. Guangdong General Hospital. Guangdong Academy of Medical Sciences. Guangzhou, Guangdong. China. ${ }^{3}$ Biostatistics Unit. Nanfang Medical University. Guangzhou, China

\begin{abstract}
Background and aims: atrophic gastritis (AG) and dysplasia are precancerous lesions, with which early surveillance of disease progression is particularly important for patients. The present study aimed to explore potential alarming signals for atrophic gastritis progression towards dysplasia.

Methods: clinical data for patients with AG in the Digestive Endoscopy Center of Guangzhou Nanfang Hospital between 2001 and 2011 were retrospectively reviewed. Survival analysis, dichotomous logistic regression analysis and rank correlation analysis were carried out.

Results: in 234 patients with atrophic gastritis, after follow up of $0.5,1,2,5$ and 10 years, the occurrence rates of dysplasia were respectively $2.3,4.4,9.6,19.3$, and $42.4 \%$. Patients with AG combined with antral ulcer or gastric angle ulcer, had a higher risk for dysplasia than patients with simple AG $(\mathrm{OR}=2.427,95 \% \mathrm{Cl} 1.069$ $\sim 5.511, \mathrm{p}=0.034 ; \mathrm{OR}=2.961,95 \% \mathrm{Cl} 1.336 \sim 6.564, \mathrm{p}=$ $0.008)$. The constituent ratios of moderate to severe dysplasia were respectively $8.6,2.7$ and $1.2 \%(p=0.000)$ in three patient groups: AG combined with antral ulcer/gastric angle ulcer $(n=255)$, antral ulcer/gastric angle ulcer alone $(\mathrm{n}=1,389)$, and AG alone $(\mathrm{n}=$ $3,106)$. The Spearman correlation coefficients between a) Hp infection; and b) atrophy, intestinal metaplasia and dysplasia were - 0.114 $(p=0.078),-0.169(p=0.009)$ and $0.064(p>0.05)$, respectively.

Conclusions: long follow-up interval and gastric ulcer may be alarming signals for the progression of AG to dysplasia. But in patients with atrophy, intestinal metaplasia or dysplasia, Hp infection may not be a risk factor for these lesions aggravated, further studies are required to confirm it.
\end{abstract}

Key words: Atrophic gastritis. Dysplasia. Helicobacter pylori. Gastric ulcer. Follow-up.

Received: 19-01-2012

Accepted: 25-06-2012

Correspondence: Yang Bai, Yali Zhang. Department of Gastroenterology. Nanfang Hospital. Southen Medical University. Guangzhou city. 510515 Guangdong Province, China.

e-mail: baiyang1030@162.com

\section{INTRODUCTION}

Atrophic gastritis (AG) is a recognized precancerous lesion. AG-related lesions (such as intestinal metaplasia and dysplasia) are risk factors for gastric cancer (GC). Studies reported that during 5-year follow-up, the annual incidence rates of GC, intestinal metaplasia, mild to moderate dysplasia and severe dysplasia were, respectively, 0.1, 0.25, 0.6 and $6 \%$ in patients with AG in Western countries (1). With the wide application of endoscopy and the improvement of health awareness, the rate of detection of AG in clinical practice is increasing. This means that an increasing number of patients with AG will bear the psychological pressure to have follow-up exams and their financial burden. Currently, no effective preventative strategies have been developed; there is only symptomatic therapy and Helicobacter pylori (Hp) eradication. During follow-up, timely and accurate assessment of disease condition and monitoring of risk factors for dysplasia are critically important for these patients. In the present study, clinical data for patients with $\mathrm{AG}$ treated at the Digestive Endoscopy Center of the Guangzhou Nanfang Hospital in the past 11 years were retrospectively analyzed and some valuable characteristics were identified. Our aim is to study the alarming signals of progression of $\mathrm{AG}$ towards worse outcome.

\section{METHODS}

\section{Inclusion criteria and histological analysis}

Data from patients treated at the Digestive Endoscopy Center of the Guangzhou Nanfang Hospital between 2001 and

Zhang Q, Lian Z, Wang L, Wang Y, Zhi F, An S, Jiang B, Zhang $Y$, Bai Y. Analysis of alarming signals for the progression of atrophic gastritis to dysplasia. Rev Esp Enferm Dig 2012;104:399-404. 


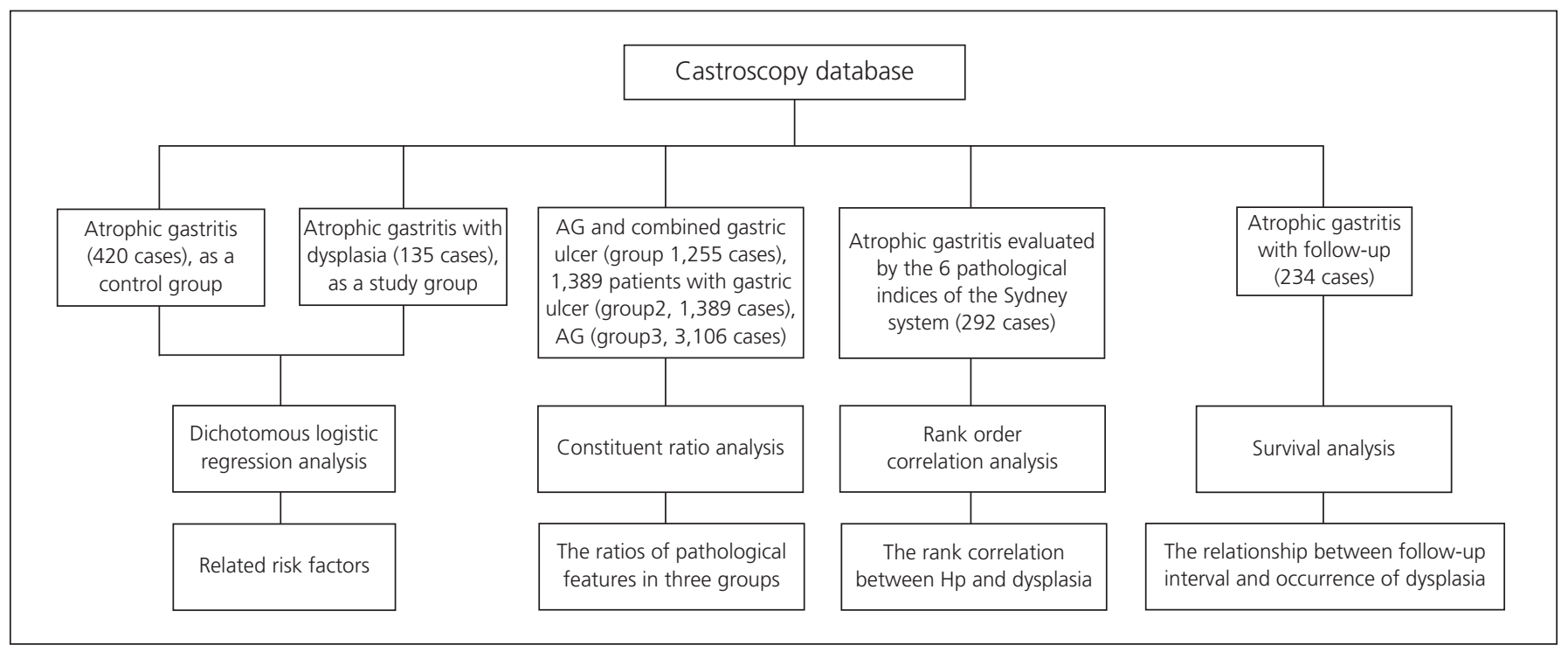

Fig. 1. Study population, statistical methodology and study aims.

2011 were retrospectively analyzed. Inclusion criteria were: 1) atrophic gastritis (AG), which is defined as loss of glandular tissue with and without metaplasia (2);2) further, the data were respectively extracted from the patients with $A G$, who were associated with dysplasia, and combined with gastric ulcer. Of these patients with AG, only part received followup, and this part of data was also extracted; and 3) gastric ulcer but not combined with atrophic gastritis (Fig. 1).

In accordance with the Updated Sydney System $(2,3)$, the degree of gastric mucosal inflammation, inflammatory activity, glandular atrophy, $\mathrm{Hp}$ infection and intestinal metaplasia were classified into four grades as follows: $0=$ none, $1=$ mild, $2=$ moderate, and $3=$ severe . And dysplasia was also classified into four grades based on the range and the degree of differentiation of dysplasia: $0=$ none, $1=$ mild, $2=$ moderate, and $3=$ severe. All samples were reviewed mainly by two pathologists, and unity discussion was performed if there was any doubt of data. The study was approved by the local Ethics Committee, and an informed consent was not considered necessary because of the observational nature of the study.

\section{Statistical analysis}

The patient data meeting the inclusion criteria above were respectively evaluated by different statistical analysis as follows (Fig. 1):

To analyze the possible risk factors for atrophic gastritis (AG), dichotomous logistic regression analysis was performed. The dependent variables were $A G$ as a control group and AG concomitant with dysplasia as a study group. The independent variables were age, gender, intestinal metaplasia, Hp infection and comorbidities including antral ulcer, gastric angle ulcer, duodenal ulcer, reflux esophagitis, bile reflux, esophageal erosion and duodenitis. As for the control group, specific sampling methods: the number of control patients for each year was three times the number of patients in the study group in each year. Then the proportion of these patients in original control patients in each year was calculated as a sampling ratio within each year. Then, sampling was done for each year based on the sampling ratio.

The three patient groups with AG and combined antral ulcer/gastric angle ulcer (group 1), with antral ulcer/gastric angle ulcer (group 2), and with simple AG (group 3), were respectively stratified according to five pathological features: inflammation or atrophy, mild to moderate dysplasia, moderate to severe dysplasia, confirmed gastric cancer and highly suspected gastric cancer. Then, constituent ratios of the pathological features were analyzed respectively in these three patient groups.

The cumulative hazard of the occurrence of dysplasia in the patients with AG was determined on the basis of the Kaplan-Meier method $(4,5)$ during follow-up. To assess the rank correlation between Hp infection and dysplasia based on the 6 pathological indices of the Sydney system, we fitted the data into the Spearman correlation analysis.

Differences were considered to be significant when the two-sided $p$ value was $<0.05$. All calculations were performed using the statistical software SPSS 13.0.

\section{RESULTS}

\section{General characteristics of patients meeting the inclusion criteria}

Between 2001 and 2011, 3,361 patients underwent gastroscopy and were confirmed as atrophic AG, of whom 
Table I. The constituent ratio of sex of different disease groups and mean age (mean \pm SD)

\begin{tabular}{lccr}
\hline & Sex & Age, mean (SD) \\
\cline { 2 - 4 } Diseases $(n)$ & Men (\%)* & Women (\%) \\
\hline Atrophic gastritis (420 patients) & $268(63.8)$ & $152(36.2)$ & $53.5(12.7)$ \\
Atrophic gastritis with dysplasia** (135 patients) & $102(75.6)$ & $33(24.4)$ & $13(21.7)$ \\
Moderate or severe dysplasia (60 patients) & $47(78.3)$ & $53(20.8)$ & $53.9(12.2)$ \\
Atrophic gastritis with gastric ulcer (255 patients) & $202(79.2)$ & $5(22.7)$ & $54.0(12.1)$ \\
Moderate or severe dysplasia (22 patients) & $17(77.3)$ & $54.4(10.8)$ \\
\hline
\end{tabular}

${ }^{*} p<0.001 . * *$ Dysplasia includes mild, moderate and severe dysplasia.

135 patients suffered AG associated with dysplasia, 234 patients received follow-up, 255 patients with AG were combined with gastric ulcer including antral ulcer or gastric angle ulcer, 292 patients with AG were evaluated by two pathologists based on the 6 pathological indices of the Sydney system. At the same time, 1,389 patients suffered gastric ulcers but not combined with atrophic gastritis (Fig. 1).

For patients with $\mathrm{AG}, \mathrm{AG}$ with dysplasia, and AG with moderate or severe dysplasia, age and gender were analyzed. The proportion of male patients was higher than female and constituent ratio of males was respectively 63.8, 75.6 and $78.3 \%$ in this three patient groups, mean ages were $53.5 \pm$ $12.7,53.9 \pm 12.2$, and $56.2 \pm 12.1$ years old, respectively (Table I).

\section{Survival analysis of AG patients with occurrence of dysplasia}

For AG patients with complete follow-up, the occurrence of dysplasia including mild, moderate and severe dysplasia was defined as an endpoint event for survival analysis. After follow up of $0.5,1,2,5$, and 10 years, the occurrence rates of dysplasia were respectively $2.3,4.4,9.6,19.3$, and 42.4 (Fig. 2).

\section{AG in combination with antral ulcer or gastric angle ulcer may be an alarming signal for the progression of atrophic gastritis towards dysplasia}

A total of 135 patients with AG and dysplasia were included as the study group. Following stratified sampling from 3,106 patients with AG but without dysplasia, 420 patients were finally included as the control group. Then dichotomous logistic regression analysis was performed. Gender, antral ulcer, and gastric angle ulcer were significantly different in the study group than in the control group ( $\mathrm{p}=0.011,0.034$, and 0.008 , respectively). These results indicated that these three factors are related to the occurrence of dysplasia in $\mathrm{AG}$ patients $(\mathrm{OR}=0.534,95 \% \mathrm{CI}$ : $0.329 \sim 0.864 ;$ OR $=2.427,95 \%$ CI: $1.069 \sim 5.511 ; 2.961$, 95\%CI: $1.336 \sim 6.564$, respectively). However, age, Hp infection and other comorbidities did not show significant differences (Table II).

In three patient groups: group 1 (AG combined with antral ulcer or gastric angle ulcer; 255 patients), group 2 (antral ulcer/gastric angle ulcer alone; 1,389 patients), group 3 (AG alone; 3,106 patients), the constituent ratios of five pathological features as mentioned above were respectively analyzed. Our findings indicated that the rate of moderate to severe dysplasia in the group 1 was significantly higher than in two other groups; the rates were 8.6, 2.7, and 1.2\%, respectively $(\mathrm{p}<0.0001)$. The rates of cancer and highly suspected cancer in the group 2 were 4.4 and $3.1 \%$, respectively, but those of were all 0\%, 0\% in the group 1 and 3 (Fig. 3).

\section{Correlations among Hp infection, chronic inflammation, atrophy, intestinal metaplasia and dysplasia}

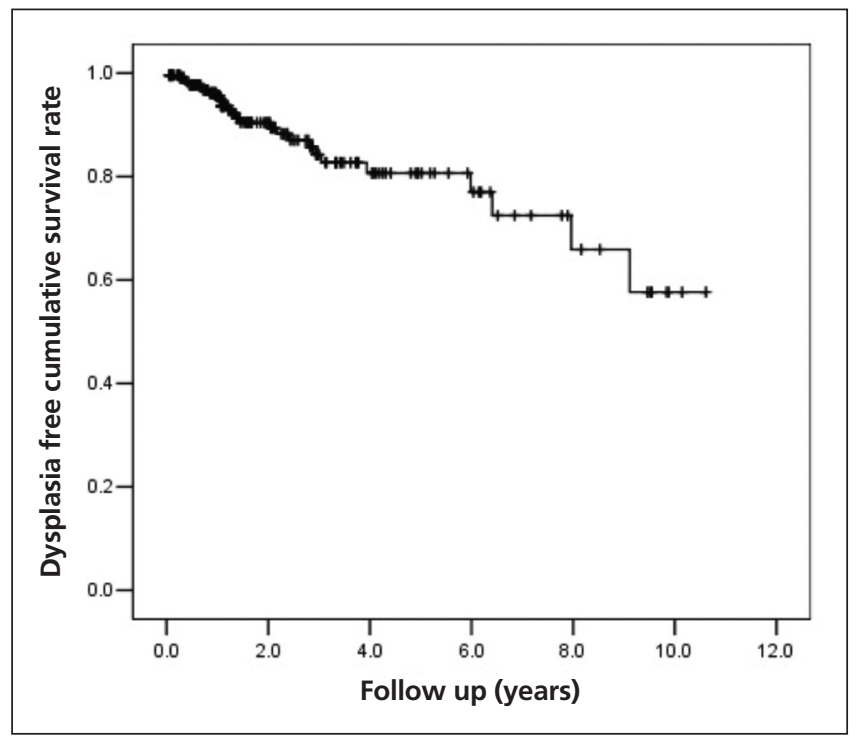

Fig. 2. Survival analysis of atrophic gastritis (AG) patients with occurrence of dysplasia as the endpoint. Cumulative dysplasia-free interval curves were drawn and analyzed using Kaplan-Meier method. Statistical significance was set at $p<0.05$. The curve showed occurrence rate of dysplasia increases gradually as follow-up time is prolonged. 
Table II. Related risk factors screening for dysplasia in dichotomous logistic regression model

\begin{tabular}{llc}
\hline & OR (IC 95\%) & $p$ \\
\hline Gender & $0.534(0.329-0.864)$ & 0.011 \\
Antral ulcer & $2.427(1.069-5.511)$ & 0.034 \\
Angular ulcer & $2.961(1.336-6.564)$ & 0.008 \\
\hline
\end{tabular}

Of 292 patients with AG evaluated according to six pathological features of The Updated Sydney System including Hp infection, chronic inflammation, inflammation activity, atrophy, intestinal metaplasia and dysplasia, rank correlation analysis was performed. Results showed that the correlationcoefficient was $0.755(\mathrm{p}<0.001)$, showing the strong correlation between atrophy and intestinal metaplasia; the correlation coefficient was $0.426(p<0.001)$, showing a positive correlation between inflammation activity and Hp infection. However, there were not significant correlations among other pathological features showed in table III in this study.

\section{DISCUSSION}

In the present study, the age of patients with AG ranged from 16 to 92 years and the mean age was $53.5 \pm 12.7$ years old. The mean age of patients developing dysplasia and moderate to severe dysplasia was $53.9 \pm 12.2$ and $56.2 \pm 12.1$ years old. Adamu et al. followed the patients aged $50 \sim 74$ years old for 5 years. Their results showed that the incidence of $A G$ in patients aged $50 \sim 54$ years old and those aged $70 \sim 74$ years old was 0.5 and $2.1 \%$, respectively. Thus, advanced age is an important risk factor of atrophy and dysplasia (6-8). In patients with AG-related diseases, the proportion of male patients is higher than that of female patients. With clinical increases in
Table III. Rank order correlation analysis performed for AG graded based on the 6 pathological indices of the Sydney system

\begin{tabular}{lcc}
\hline & $\begin{array}{c}\text { Correlation } \\
\text { coefficient }\end{array}$ & Sig. (2-tailed) \\
\hline Hp vs. chronic inflammation & 0.202 & 0.002 \\
Hp vs. inflammatory activity & 0.426 & 0.000 \\
Hp vs. atrophy & -0.114 & 0.078 \\
Hp vs. intestinal metaplasia & -0.169 & 0.009 \\
Hp vs. dysplasia & 0.064 & 0.323 \\
\hline Chronic inflammation vs. atrophy & 0.350 & 0.000 \\
Chronic inflammation vs. metaplasia & 0.231 & 0.000 \\
Chronic inflammation vs. dysplasia & 0.237 & 0.000 \\
\hline Inflammatory activity vs. atrophy & -0.111 & 0.058 \\
Inflammatory activity vs. metaplasia & -0.195 & 0.001 \\
Inflammatory activity vs. dysplasia & -0.159 & 0.007 \\
\hline Atrophy vs. intestinal metaplasia & 0.755 & 0.000 \\
Atrophy vs. dysplasia & 0.234 & 0.000 \\
Intestinal metaplasia vs. dysplasia & 0.190 & 0.001 \\
\hline
\end{tabular}

follow-up duration, the proportion of patients developing dysplasia and moderate to severe dysplasia has been increasing.

Our study showed that AG combined with gastric ulcer (especially gastric angle ulcer) is more susceptible to developing dysplasia than AG alone. Further in this study the constituent ratio of moderate to severe dysplasia in AG patients combined with antral ulcer/gastric angle ulcer was higher than in patients with gastric ulcer alone and AG alone. As shown in previous studies, in addition to the severity and distribution characteristics of atrophy and intestinal metaplasia, concomitant diseases (especially the mucosa-asso-

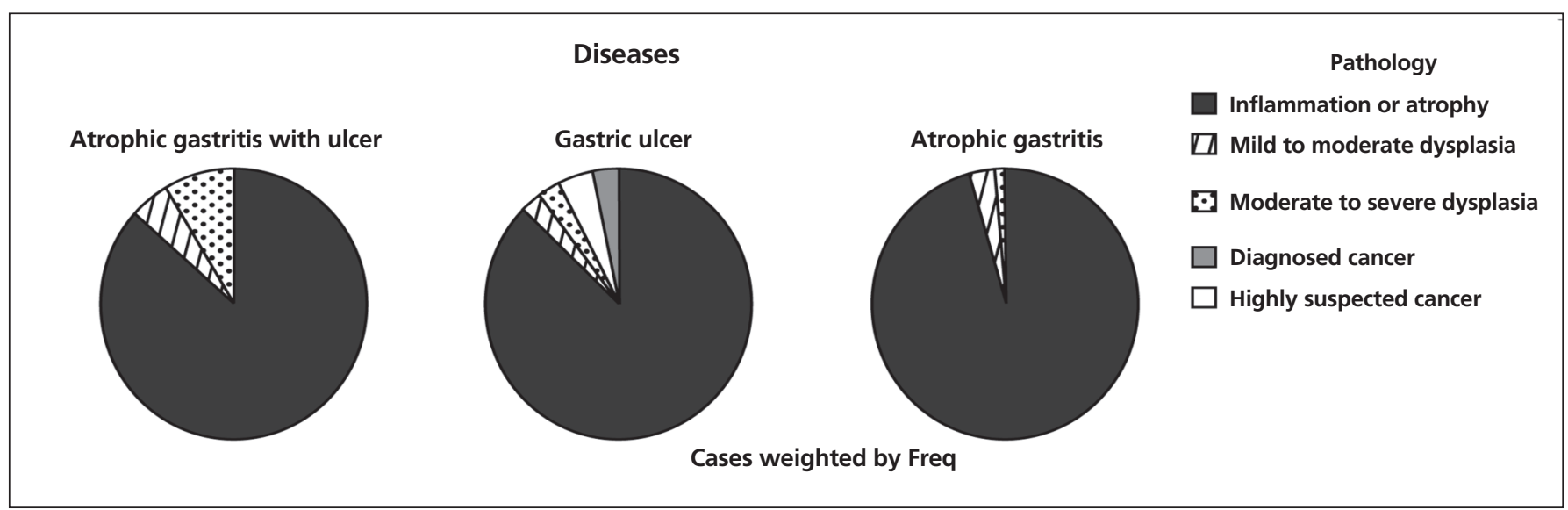

Fig. 3. The constituent ratios were analyzed based on five pathological features in three patient groups showed on the picture. The pathological indices above described: highly suspected gastric cancer was considered as gastric ulcer with few atypia cells and perishing morphogenesis under endoscope; moderate dysplasia was not included in mild to moderate dysplasia; moderate and severe dysplasia were included in moderate to severe dysplasia. 
ciated lymphoid tissue lymphoma and gastric ulcer) are risk factors for gastric cancer (9-12). There is evidence showing that type III intestinal metaplasia is frequently found in patients with delayed ulcer healing or recurrent ulcer $(75 \%)$. We speculated that gastric ulcer, type III intestinal metaplasia and $\mathrm{AG}$ are at risk for cancer, and the effect of having two or three of these factors significantly increase the risk for gastric cancer (9). There is a possible mechanism that molecular biology changes including gp130, MMP, Reg, IL-11 and IGF-1, etc., may be contributed to excessive hyperplasia of mucosal epithelial cells during the occurrence, development and outcome of diseases of gastric ulcer. Whether these changes promote the occurrence of dysplasia in the patient with atrophic gastritis combined with ulcer should be further explored (13-15). So occurrence of gastric ulcer in AG patient may be an alarming signal for the progression of atrophic gastritis towards dysplasia and AG patients with antral ulcer or gastric angle ulcer should be closely monitored in clinical work.

$\mathrm{Hp}$ infection is a risk factor for occurrence of AG. A metaanalysis showed that the relative risk of $\mathrm{Hp}$ positive patients for AG was 5.0 compared to Hp negative patients (16). However, it is unclear whether Hp infection increases the risk for disease progression in AG patients with or without intestinal metaplasia and dysplasia and whether anti-Hp therapy is necessary under this condition. In the present study, our results did not show a correlation between $\mathrm{Hp}$ infection and intestinal metaplasia or dysplasia. This suggests that $\mathrm{Hp}$ infection may be not a key risk factor of cancer development once AG, intestinal metaplasia or dysplasia has occurred. Under this condition, anti-Hp therapy might not alleviate or reverse the severity of atrophy, intestinal metaplasia and dysplasia, and then not block the progression of precancerous lesions to cancer. Wang et al. at the Queen Mary Hospital of the University of Hong Kong conducted a 7-year prospective study in which patients without precancerous lesions (AG, intestinal metaplasia and dysplasia) received anti-Hp therapy and none developed gastric cancer, but anti-Hp therapy could not block the occurrence of gastric cancer once the precancerous lesion was present (17). Precancerous lesions including AG, intestinal metaplasia and dysplasia are usually accompanied by molecular biological changes. Once the point of no return is reached, Hp eradication therapy can no longer reduce the incidence of gastric cancer. Some studies revealed that the detection rate of $\mathrm{Hp}$ infection is low in patients with $\mathrm{AG}$ and intestinal metaplasia, and the more severe the disease, the lower the detection rate (18). Thus, we speculated that Hp infection is an important risk factor for disease initiation and acts as a trigger.

Furthermore, the rank correlation analysis also showed that $\mathrm{Hp}$ infection is positively related to inflammation and chronic inflammation, and that chronic inflammation is weakly positively associated with atrophy, intestinal metaplasia and dysplasia. This may be attributed to the fact that many active substances are released during inflammation, and that the accumulation of these substances may finally cause a series of damaging events to cells such as DNA damage (19-21). Chronic inflammation may play an important role in the development of precancerous lesions $(22,23)$. Thus, in patients with precancerous lesions including AG, intestinal metaplasia or dysplasia during follow-up, $\mathrm{Hp}$ infection may not be a direct alarming signal for disease severity and/or its progression. But further basic and clinical studies are required for it.

The limitations of the present study are as follows: a) this was a retrospective study. There were some uncontrolled interfering factors which may have influenced the reliability of the results to a certain extent. However, the large sample size offset this defect partially, and b) dysplasia was categorized as mild, moderate and severe. There was inconsistency in the evaluation of mild dysplasia and different pathologists might have given different scores on any given case.

In conclusion: a) long follow-up interval and atrophic gastritis (AG) in combination with gastric ulcer (antral ulcer or gastric angle ulcer) may be potential alarming signals for dysplasia progression and should attract attention; and b) once precancerous lesions occurs, Hp infection may not directly related to disease progression towards early cancer.

The present study is a preliminary retrospective clinical study, so clinical prospective trials are further required to confirm our findings and to provide concrete guidelines for clinical practice.

\section{ACKNOWLEDGEMENT}

We thank Prof. Shengli An for the revision of the statistical analysis.

\section{REFERENCES}

1. de Vries AC, van Grieken NC, Looman CW, Casparie MK, de Vries E, Meijer GA, et al. Gastric cancer risk in patients with premalignant gastric lesions: a nationwide cohort study in the Netherlands. Gastroenterology 2008;134:945-52.

2. Dixon MF, Genta RM, Yardley JH, Correa P. Classification and grading of gastritis: The Updated Sydney System. Am J Surg Pathol 1996;20: 1161-81

3. Zhang C, Yamada N, Wu YL, Wen M, Matsuhisa T, Matsukura N. Helicobacter pylori infection, glandular atrophy, and intestinal metaplasia in superficial gastritis, gastric erosion, erosive gastritis, gastric ulcer, and early gastric cancer. World J Gastroenterol 2005;11:791-6.

4. Sauerbrei W. The use of resampling methods to simplify regression models in medical statistics. Appl Stat 1999;48:313-29.

5. Hsu CH, Long Q, Alberts DS. Estimation of colorectal adenoma recurrence with dependent censoring. BMC Med Res Method 2009;9:66.

6. Adamu MA, Weck MN, Rothenbacher D, Brenner H. Incidence and risk factors for the development of chronic atrophic gastritis: five year follow-up of a population-based cohort study. Int J Cancer 2011; 128:1652-8.

7. Weck MN, Brenner H. Prevalence of chronic atrophic gastritis in different parts of the world. Cancer Epidemiol Biomarkers Prev 2006;15:1083-94.

8. Gashi Z, Zekaj S, Haziri A, Bakalli A. The influence of the type of ulcers in the degree of atrophic gastritis. Med Arh 2011;65:20-2.

9. Silva S, Filipe MI, Pinho A. Variants of intestinal metaplasia in the evolution of chronic atrophic gastritis and gastric ulcer. A follow up study. Gut 1990;31:1097-104. 
10. Whiting JL, Sigurdsson A, Rowlands DC, Hallissey MT, Fielding JW The long term results of endoscopic surveillance of premalignant gastric lesions. Gut 2002;50:378-81.

11. Lamarque D, Levy M, Chaumette MT, Roudot-Thoraval F, Cavicchi $\mathrm{M}$, Auroux J, et al. Frequent and rapid progression of atrophy and intestinal metaplasia in gastric mucosa of patients with MALT lymphoma. Am J Gastroenterol 2006;101:1886-93.

12. Hansson LE, Nyren O, Hsing AW, Bergstrom R, Josefsson S, Chow $\mathrm{WH}$, et al. The risk of stomach cancer in patients with gastric or duodenal ulcer disease. N Engl J Med 1996;335:242-9.

13. Hodge DR, Hurt EM, Farrar WL. The role of IL-6 and STAT3 in inflammation and cancer. Eur J Cancer 2005;41:2502-12.

14. Nguyen T, Chai J, Li A, Akahoshi T, Tanigawa T, Tarnawski AS. Novel roles of local insulin-like growth factor-1 activation in gastric ulcer healing: promotes actin polymerization, cell proliferation, re-epithelialization, and induces cyclooxygenase- 2 in a phosphatidylinositol 3kinase-dependent manner. Am J Pathol 2007;170:1219-28.

15. Helander HF, Li H. Cell proliferation in the gastric epithelium of the ulcer rat. Scand J Gastroenterol 2005;40:1386-93.

16. Adamu MA, Weck MN, Gao L, Brenner H. Incidence of chronic atrophic gastritis: systematic review and meta-analysis of follow-up studies. Eur J Epidemiol 2010;25:439-48.
17. Wong BC, Lam SK, Wong WM, Chen JS, Zheng TT, Feng RE, et al. Helicobacter pylori eradication to prevent gastric cancer in a high-risk region of China. A randomized controlled trial. JAMA 2004;291:187-94.

18. Yoo JY, Kim N, Park YS, Hwang JH, Kim JW, Jeong SH, et al. Detection rate of Helicobacter pylori against a background of atrophic gastritis and/or intestinal metaplasia. J Clin Gastroenterol 2007;41:751-5.

19. Gonda TA, Tu S, Wang TC. Chronic inflammation, the tumor microenvironment and carcinogenesis. Cell Cycle 2009;8:2005-13.

20. Federico A, Morgillo F, Tuccillo C, Ciardiello F, Loguercio C. Chronic inflammation and oxidative stress in human carcinogenesis. Int J Cancer 2007;121:2381-6.

21. Kawanishi S, Hiraku Y. Oxidative and nitrative DNA damage as biomarker for carcinogenesis with special reference to inflammation. Antioxid Redox Signal 2006;8:1047-58.

22. Katsurahara M, Kobayashi Y, Iwasa M, Ma N, Inoue H, Fujita N, et al. Reactive nitrogen species mediate DNA damage in Helicobacter pylori-infected gastric mucosa. Helicobacter 2009;14:552-8.

23. Konturek PC, Rembiasz K, Konturek SJ, Stachura J, Bielanski W, Galuschka K, et al. Gene expression of ornithine decarboxylase, cyclooxygenase-2, and gastrin in atrophic gastric mucosa infected with Helicobacter pylori before and after eradication therapy. Dig Dis Sci 2003;48:36-46. 